International Journal of Pure and Applied Mathematics

Volume 112 No. 2 2017, 293-306

ISSN: 1311-8080 (printed version); ISSN: 1314-3395 (on-line version)

url: http://www.ijpam.eu

doi: 10.12732/ijpam.v112i2.7

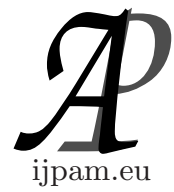

\title{
A NOTE ON THE IMPORTANCE OF EKMAN COMPATIBILITY CONDITIONS IN ROTATING BOUNDARY LAYER FLOWS
}

\author{
Satyanarayana Badeti ${ }^{1}$, Somaraju Vempaty ${ }^{2}$, S. Srinivas ${ }^{3}$ \\ ${ }^{1,3}$ Department of Mathematics \\ VIT University \\ Vellore, Tamil Nadu, INDIA \\ ${ }^{2}$ GVP - LIAS College of Engineering (Autonomous) \\ Vishakhapatnam, Andhra Pradesh, INDIA
}

\begin{abstract}
It is illustrated here, how the replacement of horizontal boundary layers (for ex. Ekman layers) in rotating flows by proper compatibility conditions makes the mathematical analysis of inviscid interior region dynamics, and vertical shear layers relatively easy. The problem of the modification of vertical free shear layers in rotating flows by the imposition of axial flow (S H Smith, QJMM, 1979, [4]) is reconsidered here for our purpose having in view its complicated mathematics and certain interesting features. In this context, we also review and discuss a few other such problems.
\end{abstract}

Key Words: rotating fluids, hydrodynamics, boundary layers, injection and suction

\section{Introduction}

The Ekman boundary layer which is a common feature of rotating flows has been found to induce an axial mass flux (known as Ekman Suction or Ekman Pumping). By this process, the Ekman layers affect significantly the transient and steady state dynamics of the rotating fluid system. The Ekman layer cannot satisfy the boundary condition on vertical velocity; it therefore gives a

Received: $\quad$ September 12, 2016

Revised: October 3, 2016

Published: $\quad$ February 1, 2017

$\S_{\text {Correspondence author }}$
(C) 2017 Academic Publications, Ltd. url: www.acadpubl.eu 
compatibility condition on axial velocity. This condition on the axial velocity is referred to as the Ekman compatibility condition on mass flow. The Ekman layers become quasi-steady with in a time of $O(1)$ and so they can be replaced conveniently by Ekman compatibility conditions in steady state as well as transient rotating flow problems. This method is found to reduce mathematical difficulties inherent in the boundary layer flows to a considerable extant [1].

The vertical boundary layers, which occur at the edge of the plates, are physically required to affect the change in angular velocity of the main body of the fluid and to balance the outflow from the boundary layer on one plate with inflow to the boundary layer on the other. The dynamics of vertical boundary layers in a rotating homogeneous fluid are best illustrated by Stewartson [2]. He considered the steady flow between two coaxial plates rotating almost as if rigid, and found that two vertical free shear layers, namely the $E^{\frac{1}{3}}$ and $E^{\frac{1}{4}}$ layers are required to complete the meridional circulation of fluid driven by Ekman layer. Stewartson did not use Ekman compatibility conditions explicitly. He expands the solutions in terms of Bessel functions and considers separable solutions, and then makes appropriate simplifications to arrive at simple integrals in complex plane reflecting the dynamics of vertical shear layers. Greenspan [3] obtained the same results by Fourier transforms and using Ekman compatibility conditions on axial velocity. The use of Ekman compatibility conditions greatly simplifies the analysis by removing the complications in mathematical analysis that arise due to the presence of singularities pertaining to Ekman layers in the Fourier transformed equations. Following Stewartson [2] and Greenspan [3], several interesting hydrodynamic and hydromagnetic vertical free shear layer problems [4] - [8] have been analyzed to understand certain aspects of astrogeophysical and geophysical flows. Hunter [9] analyzed the interior dynamics and vertical boundary layers (known as Stewartson layers) that occur in a rotating annulus due to horizontally applied temperature gradient without using the Ekman compatibility conditions. Hence, he had to solve for the Ekman extension regions to match the solutions to the side wall layer solutions. A similar problem in the presence of magnetic field [10] has been solved to lowest order with relative ease using Ekman compatibility conditions.

Though the Stewartson layers are passive in the spin-up process of a homogeneous fluid, there could be transient rotating fluid flows in which these layers could play an important part. Barcilon [11] considered such flows and used the compatibility conditions to reduce the mathematical difficulties. There are obvious advantages in replacing the Ekman layers with the compatibility conditions, while analyzing the vertical shear layers using integral transform techniques. The singularities corresponding to Ekman layers disappear from 
the solutions in the complex plane.

Smith [4] analyzed the interesting problem of modification of boundary layers (both horizontal and vertical layers) by the imposition of axial velocity in a rotating axisymmetric flow. He considered a split disc configuration and used integral transform technique to investigate the vertical shear layers. The Ekman compatibility conditions have not been used. Following Stewartson, he assumed solutions of the form $\psi=r \sum_{n=1}^{6} \int_{0}^{\infty} B_{n}(k) J_{1}(k r) e^{\alpha_{n} z} d k$, where the summation goes over all the six roots (two corresponding to the bottom layer, two for the top layer, and two for the vertical shear layers). The mathematical analysis becomes formidable. However, necessary simplifications are made to study the vertical shear layers and the integrals are reduced to simple form in the complex plane. But, replacement of Ekman layers by the compatibility conditions makes the analysis relatively simpler.

In order to illustrate the advantages of using compatibility conditions on axial flow, we consider the same problem analyzed by Smith. Thus, the present paper on free shear layers in a rotating fluid with injection and suction (also see [12]) follows the same mathematical technique as followed by Greenspan [3] to analyze the vertical boundary layer structure. Though this work does not reveal any new features different from that of Smith [4], it may be treated as a supplement/complement to Smith's work and also as a contribution to mathematical and physical fluid dynamics. It may be clearly noted at this juncture that both problems are physically same but the methods of mathematical analysis are different. It should, however, be born in our mind that the compatibility conditions are applicable for a shear layer if the thickness of the horizontal layer is much less than the shear layer thickness.

The mathematical formulation is the same as that given by Smith [4]. However, to make the paper self-contained, we shall present the mathematical formulation in $\oint$ II. The analysis of free shear layers for $E^{1 / 3} \ll R \ll E^{1 / 3}$ and $R \gg E^{1 / 3}$ is presented in $\oint I I I$ and $\oint I V$. Finally, comments and conclusions are presented in $\S \mathrm{V}$.

\section{Mathematical Formulation}

The geometry of the free shear layer situation considered here is exactly the same as that considered by Greenspan [3]. We consider a homogeneous fluid confined between two rigid parallel plates at $z=0,1$ and rotating uniformly about the axis of symmetry with angular velocity $\Omega$. An inner disc of radius $r_{0}$ 


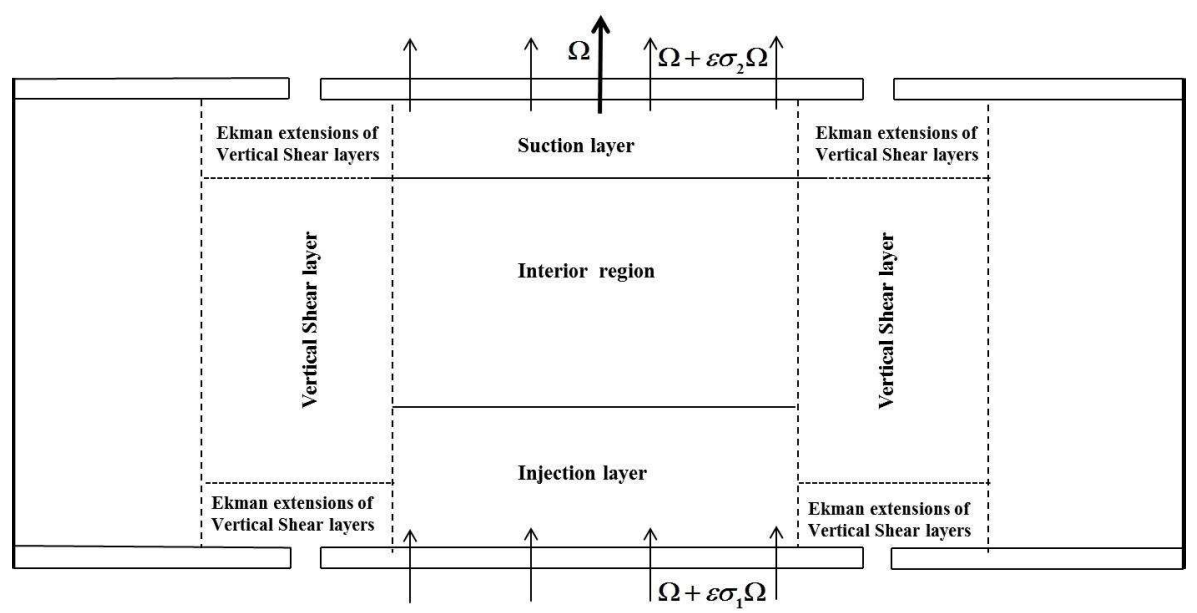

Figure 1: A diagram showing the various regions of flow in the concentric disk configuration

attached to each plate can spin independently. Departures from state of rigid body rotation are caused by small additional angular velocities $\varepsilon \sigma_{1} \Omega$ and $\varepsilon \sigma_{2} \Omega$ given to the inner discs at bottom and top plates respectively. In addition, fluid is injected with uniform velocity $U$ at the lower plate and an equal amount is sucked at the top plate (see Figure 1).

$$
\rho(\vec{V} \cdot \nabla) \vec{V}+2 \rho(\vec{\Omega} \times \vec{V})=-\nabla\left(P-\frac{1}{2} \rho \Omega^{2} r^{2}\right)+\rho v \nabla^{2} \vec{V}
$$

where $\vec{V}$ denotes velocity field, $P$ pressure field, $v$ kinematic viscosity, $\rho$ density and $r$ the radial distance. The continuity equation may be satisfied by defining a stream function $\psi$ such that the radial and axial velocity fields are respectively given by

$$
u=\frac{\partial \psi}{\partial z}, w=-\frac{1}{r} \frac{\partial(r \psi)}{\partial r}
$$

Let us introduce the dimensionless variables as follows:

$$
\begin{aligned}
\vec{V}(r, z) & =U \hat{z} \\
& +\varepsilon \Omega L\left[\frac{\partial}{\partial z^{*}}\left(\psi^{*}\left(r^{*}, z^{*}\right)\right) \hat{r}+v^{*}\left(r^{*}, z^{*}\right) \hat{\theta}-\frac{1}{r^{*}} \frac{\partial}{\partial r^{*}}\left(r^{*} \psi^{*}\left(r^{*}, z^{*}\right)\right) \hat{z}\right],
\end{aligned}
$$




$$
P=\frac{1}{2} \rho \Omega^{2} r^{2}+\rho \varepsilon \Omega^{2} L^{2} P^{*}\left(r^{*}, z^{*}\right), \quad r=L r^{*}, \quad z=L z^{*},
$$

where the parameter $\varepsilon$ is the Rossby number, which gives the ratio between inertial and Coriolis force terms. Under the assumption that the Rossby number is sufficiently small, we can linearize the governing equations, and the linearized dimensionless equations of motion in component form may be written as (dropping the asterisks)

$$
\begin{gathered}
R \psi_{z z}-2 v=-P_{r}+E\left[\nabla^{2}-\frac{1}{r^{2}}\right] \psi_{z} \\
R v_{z}+2 \psi_{z}=E\left[\nabla^{2}-\frac{1}{r^{2}}\right] v \\
R\left[\frac{1}{r}(r \psi)_{r}\right]_{z}=P_{z}+E \nabla^{2}\left[\frac{1}{r}(r \psi)_{r}\right]
\end{gathered}
$$

where $\nabla^{2}=\frac{1}{r} \frac{\partial}{\partial r}\left(r \frac{\partial}{\partial r}\right)+\frac{\partial^{2}}{\partial z^{2}}$ and subscript notation is used to denote the partial differentiation. The nondimensional parameters that appear in (3) - (5) are, number,

$$
E=\frac{\nu}{L^{2} \Omega}=\text { the Ekman number and } R=\frac{U}{L \Omega}=\text { The injection Rossby }
$$
and both of them are much less than unity.

The boundary conditions on the velocity field may be written as:

$$
\left.\begin{array}{ccrl}
v=V_{\mathbf{B}}=f(r) & =r \sigma_{1} \delta\left(1-\frac{r}{a}\right) & & \text { at } z=0 \\
v=V_{T}=g(r) & =r \sigma_{2} \delta\left(1-\frac{r}{a}\right) & & \text { at } z=1 \\
\psi & =0 & & \text { at } z=0,1
\end{array}\right\}
$$

Here $a\left(=\frac{r_{0}}{L}\right)$ is the aspect ratio and $\delta$ represents unit step function. The analysis of the problem contained in equations (3) - (5) gives us the structure of the vertical as well as horizontal boundary layers (known as injection and suction layers). Although a direct integral transform (see $[2,4]$ ) analysis of these equations is possible, we can achieve great simplification in the mathematical analysis if we replace the horizontal boundary layers by compatibility conditions (known as Ekman compatibility conditions (see $[3,5]$ )) on the interior axial flow at their outer edge. It may be noted that the injection effects are not felt by the Ekman layer for $R \ll E^{1 / 2}$ and the compatibility conditions are the same as those in the analysis of Greenspan [3]. The modification 
of interior dynamics and vertical free shear layer structure commences only for $R \gg E^{1 / 2}$. The appropriate compatibility conditions [4, 12] may be written as

$$
\left.\begin{array}{ll}
\psi=\frac{R}{2}\left(V_{B}-v_{i}\right) & \text { at } z=0 \\
\psi=0+O\left(\frac{E^{2}}{R^{3}}\right) & \text { at } z=1
\end{array}\right\}
$$

These conditions follow from the individual solutions obtained for the injection and suction layers. We shall explain the physics behind these conditions. The lower boundary layer (known as injection layer) thickens to $O\left(\frac{R^{3}}{E}\right)$ due to injection of fluid and hence it can support mass flux greater in magnitude than $E^{1 / 2}$. This induced mass flux is due to the difference in vorticity between the interior and the bottom boundary. The thickness of the top boundary layer (known as suction layer) decreases and is $O\left(\frac{E}{R}\right)$. The suction layer cannot support the mass flux greater than $O\left(E^{1 / 2}\right)$. During the transient period of the flow, the mass flux through interior and the injection boundary layer at $z=0$ is $O(R)$. As the spin-up [13] is achieved in a time $O\left(R^{-1}\right)$ the azimuthal velocity in the interior and the injection layer become the same as that of the bottom disc and the meridional flow becomes very weak, while the side wall layers can still support a mass flux $O(R)$. The compatibility conditions $(7)$ are valid in the parametric region $E^{1 / 2} \ll R \ll E^{1 / 3}$ only. When $R \gg E^{1 / 3}$, the injection boundary layer merges with the interior and there is no longer any horizontal boundary layer at $z=0$. The boundary conditions become

$$
\left.\begin{array}{cl}
\psi=0 & \text { at } z=0 \\
v=V_{B}=r \sigma_{1} \delta\left(1-\frac{r}{a}\right) & \text { at } z=0
\end{array}\right\}
$$

Now, the problem no longer involves the horizontal boundary layers, the vertical derivative terms multiplied by the Ekman number can be safely neglected. Also, since the viscous vertical shear layers are very thin, and we are mainly interested in studying their structure, we may neglect the curvature terms multiplied by the Ekman number. Eliminating pressure terms from (3) (5), the governing equations which are valid in vertical shear layer regions may be written as:

$$
\begin{gathered}
R \frac{\partial^{2} \psi}{\partial z \partial x^{2}}-2 \frac{\partial v}{\partial z}=E \frac{\partial^{4} \psi}{\partial x^{4}} \\
R \frac{\partial v}{\partial z}+2 \frac{\partial \psi}{\partial z}=E \frac{\partial^{2} v}{\partial x^{2}}
\end{gathered}
$$


where $x=a-r$. Since, the boundary layer contributions vanish at the edge of the boundary layers, the unstrected boundary layer coordinate may be allowed to range between plus and minus infinity. The problem is now amenable to Fourier transform in x-direction. We define the Fourier transform pair as,

$$
\bar{g}(\xi)=F(g(x))=\int_{-\infty}^{\infty} g(x) e^{-i \xi x} d x \text { and } g(x)=\frac{1}{2 \pi} \int_{-\infty}^{\infty} \bar{g}(\xi) e^{i \xi x} d \xi
$$

\section{Analysis of Free Shear Layers for $E^{1 / 2} \ll R \ll E^{1 / 3}$}

The equations (9) - (10) along with the boundary conditions (7) may now be written after taking Fourier transforms as

$$
\begin{gathered}
R \xi^{2} \bar{\psi}_{z}+2 \bar{v}_{z}=-E \xi^{4} \bar{\psi} \\
R \bar{v}_{z}+2 \bar{\psi}_{z}=-E \xi^{2} \bar{v}
\end{gathered}
$$

with boundary conditions

$$
\left.\begin{array}{ll}
\bar{\psi}=\frac{R}{2}\left(\bar{V}_{B}-\bar{v}_{i}\right) & \text { at } z=0 \\
\bar{\psi}=0+O\left(\frac{E^{2}}{R^{3}}\right) & \text { at } z=1
\end{array}\right\}
$$

where $\xi$ is the transformed variable and a bar is used to denote the variable in the transformed plane.

In what follows, we shall analyze the free shear layers under the assumption that $R \xi \ll 1$. This assumption means that the regions corresponding to $x>$ $O(R)$ contribute significantly to the dynamics of the problem. The equations (11) - (12) yield a second order linear ordinary differential equation in $z$ with constant coefficients

$$
\left(4-R^{2} \xi^{2}\right) \frac{d^{2} \bar{\psi}}{d z^{2}}-2 E R \xi^{4} \frac{d \bar{\psi}}{d z}-E^{2} \xi^{6} \bar{\psi}=0
$$

Equation (14) can be solved using the boundary conditions (13) available on $z$. Assuming a solution of the form $e^{p z}$ and substituting in (14) we get

$$
p_{1,2}=\frac{E R \xi^{4} \pm 2 E \xi^{3}}{\left(4-R^{2} \xi^{2}\right)}
$$


The characteristic roots given in (15) under the assumption $R \xi \ll 1$ and $R \ll E^{1 / 3}$ are $p_{1,2}= \pm \frac{E \xi^{3}}{2}$. After a considerable amount of algebra the solutions for the transformed variables $\bar{\psi}$ and $\bar{v}$ can be written as

$$
\begin{gathered}
\bar{\psi}=A(\xi) \sinh \frac{E \xi^{3}}{2}(z-1) \\
\bar{v}=-A(\xi) \xi \cosh \frac{E \xi^{3}}{2}(z-1)
\end{gathered}
$$

where $A(\xi)=\frac{-\bar{f}(\xi) R}{\left(2 \sinh \frac{E \xi^{3}}{2}+R \xi \cosh \frac{E \xi^{3}}{2}\right)}$ and $\bar{f}(\xi)=\frac{a \sigma_{1}}{i \xi}$ which is the Fourier transform of the function $V_{\mathbf{B}}=f(r)=r \sigma_{1} \delta\left(1-\frac{r}{a}\right)$ in and adjacent to the shear layer region where $r \cong a$.

Since, by the virtue of the boundary conditions (6) the interior solutions generated by the mechanical forcing on the horizontal boundaries can exist for only $x>0$ and such interior solutions are zero for $x<0$, the inversion contour should be taken beneath the origin.

Taking inverse Fourier transform of $\bar{\psi}$ and $\bar{v}$, the solutions for $\psi$ and $v$ in the integral form may be written as

$$
\begin{gathered}
\frac{\psi}{a \sigma_{1}}=\frac{-R}{2 \pi i} \int_{-\infty}^{+\infty} \frac{e^{i x \xi} \sinh \frac{E \xi^{3}}{2}(z-1)}{\xi\left(2 \sinh \frac{E \xi^{3}}{2}+R \xi \cosh \frac{E \xi^{3}}{2}\right)} d \xi \\
\frac{v}{a \sigma_{1}}=\frac{R}{2 \pi i} \int_{-\infty}^{+\infty} \frac{e^{i x \xi} \cosh \frac{E \xi^{3}}{2}(z-1)}{\left(2 \sinh \frac{E \xi^{3}}{2}+R \xi \cosh \frac{E \xi^{3}}{2}\right)} d \xi
\end{gathered}
$$

Since $E^{1 / 2} \ll R \ll 1$ and $R \xi<1$, the second term in the denominator of $A(\xi)$ is negligible compared to the first term. For $E^{1 / 2} \ll R \ll E^{1 / 3}$ the singularities of the integrals of (18) - (19) are given by

$$
\sinh \frac{E \xi^{3}}{2}=0 \text { leading to } \xi_{n}=\left(\frac{2 n \pi i}{E}\right)^{1 / 3} \text { for } n=1,2,3 \ldots \infty
$$

These simple isolated poles $\xi_{n}$ will give rise to Stewartson $E^{1 / 3}$ layer. In addition, $\sinh \frac{E \xi^{3}}{2}$ term can become very small in a much thicker layer when $\frac{E \xi^{3}}{2} \approx 0$ giving rise to distinct poles at $\xi=0$ and $\xi= \pm i\left(\frac{R}{E}\right)^{1 / 2}$. Note that 
the pole at $\xi=0$ gives the interior solution. It may be seen that the expression for $\psi$ does not contain the pole at $\xi=0$ because of the presence of the term $\sinh \frac{E \xi^{3}}{2}(z-1)$ in the numerator, while the expression for $v$ has a simple pole at $\xi=0$. This immediately predicts that there is no $O(R)$ mass flux in the interior. And the poles $\xi= \pm i\left(\frac{R}{E}\right)^{1 / 2}$ give rise to $\left(\frac{E}{R}\right)^{1 / 2}$ layer, which is a modified outer Stewartson layer. This layer can occur for $R \gg E^{1 / 2}$. As $R \rightarrow E^{1 / 2}$ this layer becomes the familiar Stewartson $E^{1 / 4}$ layer. The residue calculation now yields the following results for $\psi$ and $v$ :

$$
\begin{aligned}
& \frac{v}{a \sigma_{1}}=1-\frac{x}{|x|} \frac{1}{2} \exp \left[-\left(\frac{R}{E}\right)^{1 / 2}|x|\right] \\
& -\frac{2^{-2 / 3} R}{3 E^{1 / 3}} \frac{x}{|x|} \sum_{n=1}^{\infty}(-1)^{n} \frac{\cos (n \pi(z-1))}{(n \pi)^{2 / 3}} G_{n}, \\
& \frac{\psi}{a \sigma_{1}}=\frac{-R}{4}(z-1) \frac{x}{|x|} \exp \left[-\left(\frac{R}{E}\right)^{1 / 2}|x|\right] \\
& +\frac{R}{6 \pi} \frac{x}{|x|} \sum_{n=1}^{\infty}(-1)^{n} \frac{\sin (n \pi(z-1))}{n} H_{n},
\end{aligned}
$$

where

$$
\begin{gathered}
G_{n}=\exp \left(-\beta_{n}|x|\right)-2 \exp \left(-\frac{1}{2} \beta_{n}|x|\right) \cos \left(\frac{\sqrt{3}}{2} \beta_{n}|x|-\frac{\pi}{3}\right) \\
H_{n}=\exp \left(-\beta_{n}|x|\right)+2 \exp \left(-\frac{1}{2} \beta_{n}|x|\right) \cos \left(\frac{\sqrt{3}}{2} \beta_{n}|x|\right) \text { and } \beta_{n}=\left(\frac{2 n \pi}{E}\right)^{1 / 3} .
\end{gathered}
$$

The solutions (20) and (21) are only to the lowest order, and are the same as those obtained by Smith [4]. There are two side wall layers namely, an inner layer of thickness $O\left(E^{1 / 3}\right)$ and an outer layer of thickness $O\left(\frac{E}{R}\right)^{1 / 2}$ which is the modified $O\left(E^{1 / 4}\right)$ layer. The thickness of this layer agrees with the finding of Barcilon [11] that the outer Stewartson layer has a thickness equal to the distance through which vorticity can diffuse in spin-up time, which in our case is $O\left(R^{-1}\right)$. Once the spin-up process is completed, steady state is reached and 
the azimuthal velocity in the interior becomes the same as that of the bottom plate as shown by (20). As a result the Ekman suction becomes is very weak and is less than $O(R)$. However, because of the differential rotation between the thicker $O\left(\frac{E}{R}\right)^{1 / 2}$ layer and the bottom plate, and because of the thickness of the injection layer is greater than $E^{1 / 2}$ there exists an axial mass flux of $O(R)$ from $\left(\frac{E}{R}\right)^{1 / 2}$ layer into the injection region as shown by (21). The mass flux $O(R)$ is closed through the corner region, the $\left(\frac{E}{R}\right)^{1 / 2}$ layer and the $E^{1 / 3}$ layer. The suction layer extension does not support this mass flux, since its thickness is very small compared to injection layer. It is clearly seen from (21) that $\psi=0$ at $z=1$. The circulation pattern is shown in Figure (2).

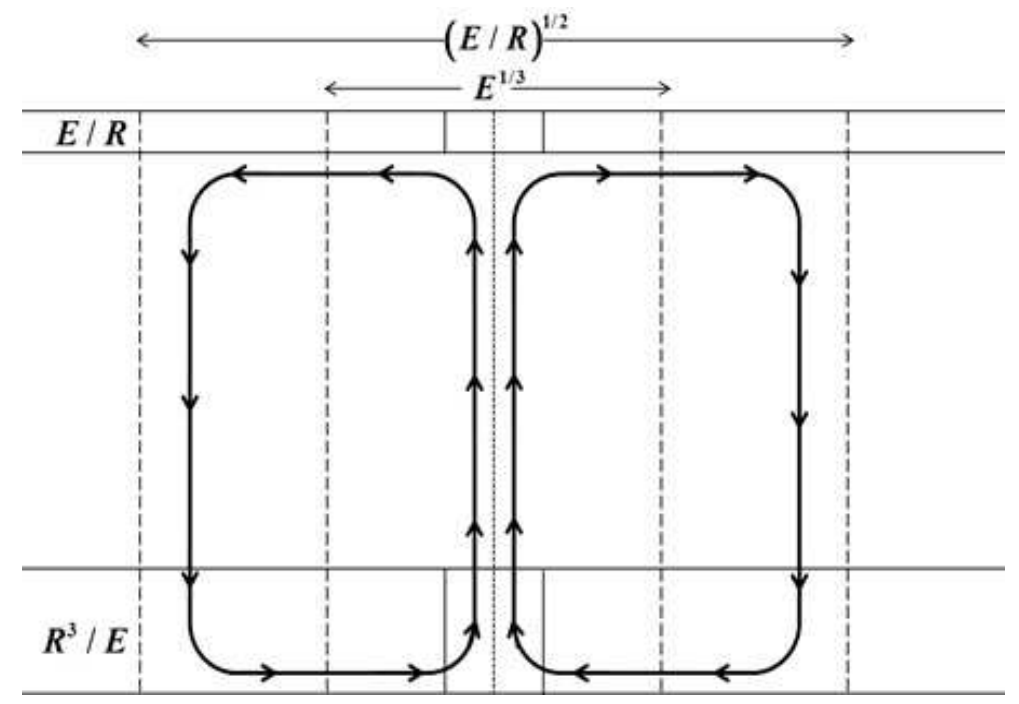

Figure 2: A schematic diagram of $O(R)$ mass flux circulation when $E^{1 / 2} \ll R \ll E^{1 / 3}$

\section{Analysis of Free Shear Layers for $R \gg E^{1 / 3}$}

It is seen that as $R \rightarrow E^{1 / 3}$, both the vertical boundary layers found for $E^{1 / 2} \ll R \ll E^{1 / 3}$ merge together. From (4) we see that injection and viscous 
terms balance each other to give rise to a single vertical boundary layer of thickness $O\left(\frac{E}{R}\right)^{1 / 2}$. For $R \gg E^{1 / 3}$ the $\frac{R^{3}}{E}$ injection layer merges with interior and there is no longer any horizontal boundary layer at $z=0$. In what follows, we shall analyze the free shear layers for $R \gg E^{1 / 3}$ under the assumption that $R \xi \gg 1$.

The characteristic roots $(15)$ for $R \gg E^{1 / 3}$ under the assumption $R \xi \gg 1$ will become $p_{1,2}=-\frac{E \xi^{2}}{R},-\frac{E \xi^{2}}{R}$ (the roots are equal). Thus, the boundary layer thickness is $O\left(\frac{E}{R}\right)^{1 / 2}$. Therefore, the solution for $\bar{\psi}$ can be written as

$$
\bar{\psi}=\left(c_{1}+c_{2} z\right) \exp \left(\frac{-E \xi^{2} z}{R}\right)
$$

The corresponding boundary conditions for the case $R \gg E^{1 / 3}$ are given in (8). In the transformed plane these boundary conditions are

$$
\left.\begin{array}{cc}
\bar{\psi}=0 & \text { at } z=0 \\
\bar{v}=a \sigma_{1} \bar{\delta}(x)=\frac{a \sigma_{1}}{i \xi} & \text { at } z=0
\end{array}\right\}
$$

From (22), (23) and (11), the expressions for $\bar{\psi}$ and $\bar{v}$ are

$$
\begin{gathered}
\frac{\bar{\psi}}{a \sigma_{1}}=\frac{2 E}{i \xi R^{2}} z \exp \left(-\frac{E \xi^{2}}{R} z\right) \\
\frac{\bar{v}}{a \sigma_{1}}=\frac{1}{i \xi} \exp \left(-\frac{E \xi^{2}}{R} z\right)
\end{gathered}
$$

Now, taking inverse Fourier transform of $\bar{\psi}$ and $\bar{v}$, the solutions for $\psi$ and $v$ are (see Campbell and Foster [14])

$$
\begin{gathered}
\frac{\psi}{a \sigma_{1}}=\frac{E z}{R^{2}} \operatorname{Erf}\left(\frac{|x|}{2} \sqrt{\frac{R}{E z}}\right) \\
\frac{v}{a \sigma_{1}}=\delta(x)-\frac{1}{2} \frac{x}{|x|} \operatorname{Erfc}\left(\frac{|x|}{2} \sqrt{\frac{R}{E z}}\right)
\end{gathered}
$$

Thus for $R \gg E^{1 / 3}$ the $E^{1 / 3}$ layer and $\left(\frac{E}{R}\right)^{1 / 2}$ layer merge together to form a single parabolic viscous boundary layer of thickness $O\left(\frac{E}{R}\right)^{1 / 2}$. The solution 
(27) corresponds to the governing equation $R v_{z}=E v_{x x}$ in the boundary layer region. It is seen from (26) that at the edge of the layer, there is a radial flow into the boundary layer. Therefore, a separate inviscid region occurs adjacent to the boundary layer to feed this $O\left(\frac{E}{R^{2}}\right)$ flux. But, this interior solution is forced by the vertical shear layer and so occurs on both sides of the discontinuity at $x=0$ (The applied mechanical forcing induces only a vertical mass flux $O(E)$ in the interior for $x>0$ only). Actually, this parabolic shear layer cannot satisfy the boundary condition on $\psi$ and so gives a compatibility condition on $\psi$. Further, a sublayer should occur at $z=1$ to satisfy the boundary condition on $\psi$ at $z=1$. The fluid circulation pattern is shown in Figure (3).

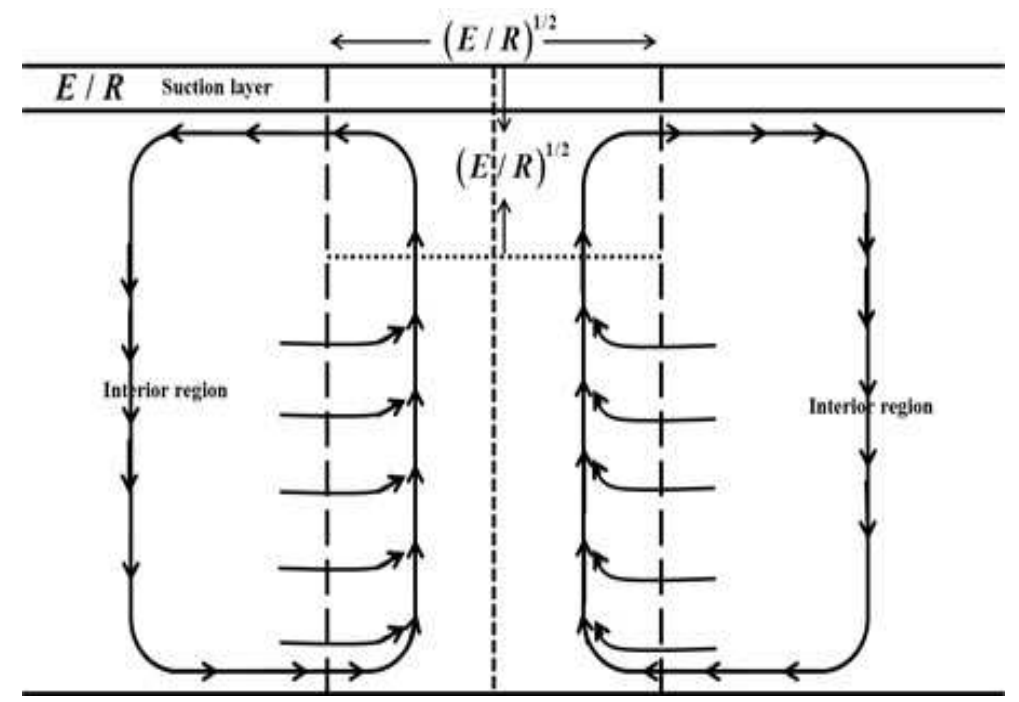

Figure 3: A schematic diagram of $O(R)$ mass flux circulation when $R \gg E^{1 / 3}$

\section{Conclusions}

As mentioned in the introduction, the boundary layer problem considered here was originally considered and analyzed by Smith [4] without recourse to compatibility conditions. The foregoing analysis of the problem in $\S I I I$ and $\S I V$ illustrates how the use of compatibility conditions, when they are applicable, reduce the mathematical complexities inherent in rotating boundary layer problems. 
It is especially true, while using integral transform methods for mathematical analysis. However, there may be some cases where the vertical shear layer may have the same thickness as that of both or one of the horizontal layers. In such cases, other simplifications such as

1. The horizontal boundary layers may become irrelevant to lower order (see $[15])$,

2. One of the horizontal layers may merge with the interior as in the present problem,

may occur to obtain the boundary conditions on $z$. It is of interest to note that in this problem, the interior cannot be completed for $R \gg E^{1 / 3}$ unless the vertical shear layer is examined.

\section{References}

[1] H P Greenspan and L N Howard, On a time dependent motion of a rotating fluid, $J$. Fluid Mech., 17, (1956), 385-404.

[2] K Stewartson, On almost rigid rotations, J. Fluid Mech., 3 (1957), 17-26.

[3] H P Greenspan, The Theory of Rotating Fluids, Cambridge University Press, Cambridge (1956).

[4] S H Smith, The modification of boundary layers by the imposition of an axial velocity within a rotating fluid, Q. J. Mech. Appl. Math, 32, (1979), 135-148.

[5] S Vempaty and D E Loper, Hydromagnetic Free Shear Layers in a rotating flow, $Z$. Angew. Math. Phys., 29 (1978), 450-461.

[6] R Hollerbach, Magnetohydrodynamic shear layers in a rapidly rotating plane layer, Geophys. Astrophys. Fluid Dynamics, 82 (1996), 237-253.

[7] S Vempaty and P Satyamurthy, Hydromagnetic free shear layers in rotating flows with magnetic field perpendicular to rotation vector and shear layers, Z. Angew. Math. Mech., 83 (2003), 321-332.

[8] A M Soward and E Dorney, Shear layers in Magnetohydrodynamic spherical Couette flow with conducting walls, J. Fluid Mech., 645 (2010), 145-185.

[9] C Hunter, The axisymmetric flow in a rotating annulus due to horizontally applied temperature gradient, J. Fluid Mech., 27 (1967), 753-778.

[10] S Vempaty and P Satyamurthy and R Balasubramanian, Hydromagnetic flow in a wall heated rotating annulus, $Z$ Angew Math Mech., 80 (2000), 273-281.

[11] V Barcilon, Stewartson layers in transient rotating fluid flows, J. Fluid Mech., 33 (1968), 815-825.

[12] S Vempaty, Effect of normal blowing on the hydrodynamic flow in a rotating cylindrical container, Indian J. Pure Appl. Math., 22 (1991), 689-702. 
[13] S H Smith, Note on boundary layer pumping, Q. J. Mech. Appl. Math, 34, (1981), 179-186.

[14] G A Campbell and R N Foster, Fourier Integrals for Practical Applications, Van Nostrand Company, (1948).

[15] V Barcilon and J Pedlosky, Linear theory of rotating stratified rotating fluid, J. Fluid Mech., 29 (1967), 1-16. 